\title{
Fault Detection and Isolation with Fluid Mechanics Constraints for Cryogenic Combustion Bench Cooling Circuit
}

\author{
C. Sarotte ${ }^{1}$, J. Marzat ${ }^{2}$, H. Piet Lahanier ${ }^{3}$, M. Galeotta ${ }^{4}$ and G. Ordonneau ${ }^{5}$ \\ 1,2,3,5 DTIS, ONERA, Université Paris-Saclay, F-91123 Palaiseau, France \\ camille.sarotte@onera.fr \\ julien.marzat@onera.fr \\ helene.piet-lahanier@onera.fr \\ gerard.ordonneau@onera.fr \\ ${ }^{4}$ CNES, Paris, France \\ marco.galeotta@cnes.fr
}

\begin{abstract}
This paper presents the design of a Fault Detection and Isolation scheme to improve the reliability of a cryogenic engine test bench operation, focusing specifically on its cooling circuit. The proposed fault detection consists in an extended unknown input observer, a cumulative sum algorithm and an exponentially moving average chart. A dynamic parity space approach is then proposed to isolate one or two simultaneous faults in the cooling circuit. The initial system model, for each line composing the cooling circuit, is augmented with constraints based on the mass flow rate continuity and the energy conservation for the overall system. Time delays in the transients are accounted for by recursive equations over a sliding window. The method allows settling adaptive thresholds that avoid pessimistic decision about the continuation of tests while detecting and isolating faults in the transient and permanent states of the system. The model structure and the estimation method were validated on the real Mascotte test bench (ONERA/CNES) data. The fault detection and isolation scheme was validated in realistic simulations.
\end{abstract}

\section{INTRODUCTION}

The need for increased launch safety and launcher's engines reliability leads to the development of health monitoring systems. The monitoring of test benches and engines is a major challenge in the development and integration of new propulsion systems for rockets, including reusable ones. The experience acquired during the years of Ariane 5 system's exploitation has pointed out the complexity of the implementation of cryogenic propulsive systems as well as the necessity

\footnotetext{
Camille Sarotte et al. This is an open-access article distributed under the terms of the Creative Commons Attribution 3.0 United States License, which permits unrestricted use, distribution, and reproduction in any medium, provided the original author and source are credited.
}

to get a specialized expertise on the physical phenomenon. In terms of traditional engines the objective is the improvement and the reliability of the implementation (see (Iannetti, Marzat, Piet-Lahanier, Ordonneau, \& Vingert, 2014)). The methods commonly used nowadays consist in checking if the measured values exceed a predetermined redline during the test. This type of approach requires selecting multiple thresholds for each test, which can be complex and can possibly lead to a pessimistic attitude about the continuation of the test. To handle emergency situations arising from actuator failures that can affect the engine performances, the failure should be detected quickly, then isolated and its causes should be identified. For that purpose, Fault detection and isolation (FDI) methods have been developed to evaluate failures and take a decision using all available information with the help of explicit or implicit models. The most common modelbased approach makes use of observers to generate residuals. Faults are detected by setting a fixed or variable threshold on each residual signal as in (Basseville, Nikiforov, et al., 1993). Those FDI methods assume that the mathematical model used is representative of the system dynamics. This is challenging in practice because of the presence of modeling uncertainties and unknown disturbances. To tackle the problem of unknown disturbances, a simple class of full order observers for linear systems with unknown inputs can be used. It consists in a coordinate system transformation that decouples the disturbance effect on the system outputs. The observer resulting from such an approach is called Unknown input observer (UIO) (see for example (Darouach, Zasadzinski, \& Xu, 1994)). In the case of non-linear systems one of the developed techniques is to linearize and design an Extended unknown input observer (EUIO) as described in (Witczak, 2007).

The parity space-based fault detection approach is also one of the most common approaches to residual generation by using 
parity relations. Those relations are rearranged direct inputoutput model equations subjected to a linear dynamic transformation. The design freedom obtained through the transformation can be used to decouple disturbances and improve fault isolation. Parity space approaches have been proved to be structurally equivalent to the observer-based though the design procedures differ (Gertler, 2017). However, the parity space methodology using the temporal redundancy has its specific advantages, especially for discrete-time systems. For example, a way to overcome time delays is to use recursion over a sliding window (Wang, Tian, Shi, \& Weng, 2010). In most existing works, the projection matrix for a parity check is chosen arbitrarily (Gao, Cecati, \& Ding, 2015). Some recent works have proposed a new parity space approach including methods to design the projection matrix for realistic situations considering the general system including system and measurement noises and accounting for actuator and sensor faults, simultaneously (Kim \& Lee, 2005). However, this work assumes that the fault is constant on the interval of parity spaces order. Another method to isolate faults is to establish a relationship between parity-space based fault detection and a minimization problem (Zhong, Song, \& Ding, 2015). In this paper a FDI method for the cooling circuit of a cryogenic combustion bench, Mascotte (CNES/ONERA), is studied. This bench has been developed to study heat transfers in the combustion chamber and jet separation in nozzles in the same conditions as for Vulcain 2 motor. Due to high combustion temperatures and high heat transfer rates from the hot gases to the chamber wall, the thrust chamber cooling requires major design consideration. The optimization of the chamber pressure value for a high-performance engine system is mostly limited by the capacity and efficiency of the chamber cooling system. In turn, chamber pressure will affect other design parameters such as nozzle expansion area ratio, propellant feed pressure, and weight. The method proposed here is based on a physical model giving the output pressure and mass flow rate of the cooling circuit (Section 2). The fault has its own known dynamic which allows us to use direct fluid mechanics constraints. To generate residuals we estimate the state with the help of an EUIO (Section 3). The proposed method permits to deal with measurement and process noises, as well as unknown inputs without solving minimization problems. Then the residuals are analyzed with a Cumulative sum (CUSUM) algorithm using an Exponentially weighted moving average (EWMA-C) chart to detect a mean shift (Jiang, Shu, \& Apley, 2008; Ryu, Wan, \& Kim, 2010; Basseville et al., 1993) and a recursive parity check for the overall system achieves fault isolation. This complete FDI method coupling a model-based observer and a parity space approach permits to localize an actuator additive fault in spite of unmeasured information and have been validated with offline tests based on real data and realistic Carins (CNES simulation software) simulations.

\section{SYSTEM DESCRIPTION}

The cryogenic combustion bench Mascotte (Figure 1) performs an oxygen / hydrogen operation with pressures and mass flow rates comparable to an injection element of the Vulcain 2 engine. The cooling system makes use of water as coolant. This circuit permits to cool the ferrules of the combustion chamber and an axisymetric nozzle. The detection of a leak or an obstruction is a critical safety task for the bench operation. The water cooling circuit consists in different pipes sections with multiple pressure release valves and a tank at the inlet. The available measurements are pressure, mass flow and temperature. Sections are separated by sliding valves with additional pressure measurements.

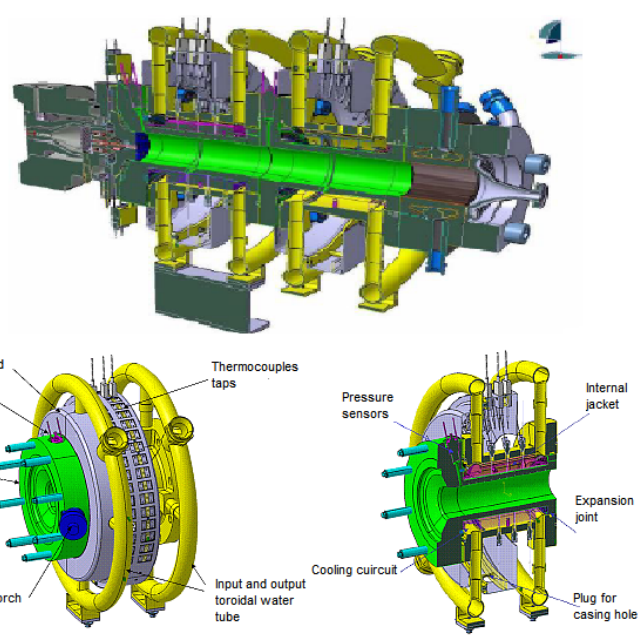

Figure 1. Mascotte test bench - (ONERA - CNES)

The circuit can be modeled by a succession of cavities defined in pressure and temperature linked by orifices where friction forces and heat flux exchanges are taken into account, see (Iannetti et al., 2014). A pressure regulator (actuator) permits to regulate the input cavities pressures of the water cooling circuit.

The performances of the algorithms are evaluated by two means. The first one is based on off-line tests realized with real measurements of the project Conforth (CNES/ONERA). During those trials, pressures (cavities 1 and 2), temperatures (cavities 1,2 and wall) and output mass flow rate have been recorded. The second one is based on a simulation software, Carins (CNES). The simulator an identical bench permits to simulate failures for the evaluation of performances with various faults.

\subsection{Model of the cooling circuit}

In this section we denote $\dot{m}$ the mass flow rate $(\mathrm{kg} / \mathrm{s}), \rho$ the density $\left(\mathrm{kg} / \mathrm{m}^{3}\right), S$ the surface $(\mathrm{m}), c$ the velocity of sound $(\mathrm{m} / \mathrm{s}), u$ the fluid velocity $(\mathrm{m} / \mathrm{s}), P$ the pressure $(\mathrm{Pa}), D$ the orifice diameter $(\mathrm{m}), D_{h}$ the hydraulic diameter $(\mathrm{m}), L$ the 
length (m), $\mu$ the dynamic viscosity (Pa.s) and $V$ the volume $\left(\mathrm{m}^{3}\right), d_{t}$ the time step $(\mathrm{s})$.

The tests have been performed for a particular configuration with a two-dimensional nozzle and a visualization window located at the nozzle throat. The nozzle cooling part of Mascotte cooling circuit is modeled by a succession of cavities and orifices in parallel (Figure 2):

- The total pressure after the sphere is of 39 bars (element 100 on the synoptic),

- The part before the visualization window composed of 3 lines with a mass flow rate of $2.5 \mathrm{~L} / \mathrm{s}(94)$,

- The part cooling the walls before the visualization window with a mass flow rate of $0.5 \mathrm{~L} / \mathrm{s}(96,97,57,58)$,

- The part cooling the bottom before the visualization window with a mass flow rate of $1.5 \mathrm{~L} / \mathrm{s}(98,59)$,

- The part after the visualization window composed of 4 lines with a mass flow rate of $0.75 \mathrm{~L} / \mathrm{s}$ each (102).

- The part cooling the walls after the visualization window with a mass flow rate of $0.2 \mathrm{~L} / \mathrm{s}(109,110,79,80)$,

- The part cooling the bottom after the visualization window with a mass flow rate of $0.2 \mathrm{~L} / \mathrm{s}(111,90)$,

- The line cooling the helium throat with a mass flow rate of $0.15 \mathrm{~L} / \mathrm{s}$. An obstruction has been simulated on the part

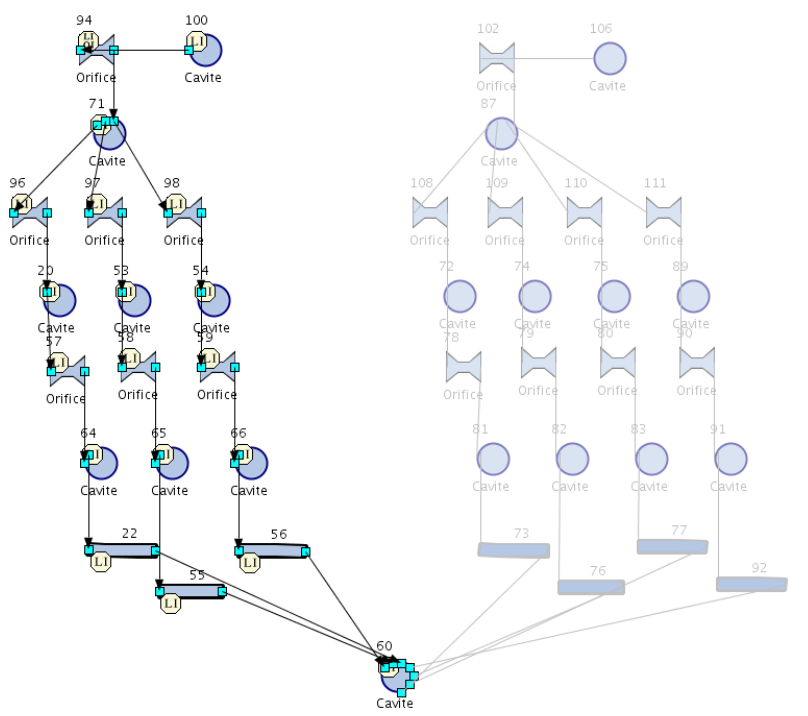

Figure 2. Mascotte cooling circuit - Carins

before the visualization window of the cooling circuit. The faults have been simulated for each case on one or two different lines $(96,97$ or 98$)$.

For our model, we consider 3 input cavities $(20,53,54)$, giving input pressures, linked by orifices $(57,58,59)$, giving the mass flow rates, to 3 output cavities $(64,65,66)$, giving the output pressures. The flow is assumed to stay monophasic, is ideal (no force due to viscosity acts) and incompressible following Euler equations. The cavity section is assumed constant and the velocity of sound is defined as for an isentropic reaction in the orifice. We assume that the fluid flow velocity is small in comparison to the velocity of sound.

The flow crossing cavities respects the mass balance equation, after integrating this equation along the cavity length, we obtain:

$$
\frac{\partial P}{\partial t}=\frac{c^{2}}{V}\left(\dot{m}_{e}-\dot{m}_{s}\right)
$$

with $\dot{m}_{e}$ and $\dot{m}_{s}$ respectively the input and output mass flow rates. The flow crossing the orifice between the two cavities respects the momentum balance equation with friction forces, expressed with the Darcy-Weisbach and Blasius equations for moderate turbulent flows in a smooth pipe (Nakayama, 1998). After integrating this equation along the orifice length we obtain:

$$
\frac{1}{S^{2}} \frac{\partial \dot{m}}{\partial t}+\frac{\Delta P}{V}=-\lambda_{p d c} \frac{L}{D_{h}} \frac{\dot{m}^{2}}{2 \rho V S^{2}}
$$

with $\lambda_{p d c}$ the friction coefficient. The model for each line of this part of the cooling circuit is then:

$$
\left\{\begin{array}{c}
\frac{\partial \dot{m}_{e}}{\partial t}=\theta_{1} \dot{m}_{e}^{\frac{7}{4}}-\theta_{2} \Delta P \\
\frac{\partial P}{\partial t}=-\theta_{3} \Delta \dot{m}
\end{array}\right.
$$

with $\theta_{1}:=-0.316\left(\frac{4}{\pi D \mu}\right)^{-\frac{1}{4}} \frac{L}{D_{h}} \frac{1}{2 \rho V}, \theta_{2}:=\frac{S^{2}}{V}, \theta_{3}:=\frac{c^{2}}{V}$

For a circular pipe, the hydraulic diameter $D_{h}$ is equal to the diameter of the pipe.

The previous model (Iannetti et al., 2014) presented approximations in the transient of the test bench inducing the presence of a pressure difference square root, moreover, the mass flow rate dynamics was not modeled. The model presented here permits to determine the pressure but also the mass flow rate and it is now possible to model their evolution during the motor speed transients. The model has been studied and validated in (Sarotte et al., 2018).

For improving readability, the synoptic is simplified as in Figure 3 with the input cavities (1,2 and 3$)$ and the output cavities $(4,5$ and 6$)$ linked by orifices.

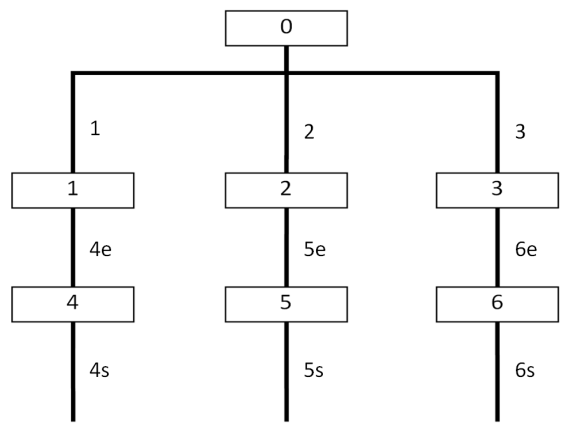

Figure 3. Mascotte cooling circuit - Conforth configuration Carins 


\section{FAUlt detection AND isolation}

\subsection{Unknown Input Observer design}

The first step is to design an observer to estimate the state in the presence of unknown inputs. The system (3), can be rewritten as a linear time-varying system with an unknown input by linearizing around a steady-state equilibrium trajectory corresponding to the mass flow trajectory to estimate the engine speed transients. Then, the system can be transformed into an equivalent discrete-time state space system with an Euler explicit scheme:

$$
\left\{\begin{array}{c}
X_{k+1}=A_{k}(\bar{X}) X_{k}+B U_{k}+E D_{k} \\
Y_{k+1}=C X_{k+1}
\end{array}\right.
$$

where $X_{k}$ is the state vector, $Y_{k}$ is the measured output vector, $U_{k}$ is the known measured input vector, $D_{k}$ is the unknown input vector and $\bar{X}$ is the equilibrium trajectory.

$$
\begin{gathered}
X_{k}:=\left[\begin{array}{c}
\dot{m}_{4, e, k} \\
P_{4, k} \\
\dot{m}_{5, e, k} \\
P_{5, k} \\
\dot{m}_{6, e, k} \\
P_{6, k}
\end{array}\right], Y:=\left[\begin{array}{lll}
P_{4, k} & P_{5, k} & P_{6, k}
\end{array}\right]^{T}, \\
U:=\left[\begin{array}{lll}
P_{1, k} & P_{2, k} & P_{3, k}
\end{array}\right]^{T}, D:=\left[\begin{array}{lll}
\dot{m}_{4, s, k} & \dot{m}_{5, s, k} & \dot{m}_{6, s, k}
\end{array}\right]^{T}
\end{gathered}
$$

Where $\dot{m}_{4,5,6, e}$ are input mass flow rates, $P_{4,5,6}$ are the pressure of output cavities, $P_{1,2,3}$ are the pressure of input cavities and $\dot{m}_{4,5,6, s}$ are output mass flow rates.

With $A_{k}$ the state matrix, $B$ the known input distribution matrix, $E$ the unknown input distribution matrix et $C$ the output distribution matrix.

$$
\begin{aligned}
& A_{k}:=\left[\begin{array}{ccc}
A_{1, k} & 0_{2,2} & 0_{2,2} \\
0_{2,2} & A_{2, k} & 0_{2,2} \\
0_{2,2} & 0_{2,2} & A_{3, k}
\end{array}\right], B:=\left[\begin{array}{ccc}
B_{1} & 0_{1,2} & 0_{1,2} \\
0_{1,2} & B_{2} & 0_{1,2} \\
0_{1,2} & 0_{1,2} & B_{3}
\end{array}\right] \\
& E:=\left[\begin{array}{ccc}
E_{1} & 0_{1,2} & 0_{1,2} \\
0_{1,2} & E_{2} & 0_{1,2} \\
0_{1,2} & 0_{1,2} & E_{3}
\end{array}\right], C:=\left[\begin{array}{cccccc}
0 & 1 & 0 & 0 & 0 & 0 \\
0 & 0 & 0 & 1 & 0 & 0 \\
0 & 0 & 0 & 0 & 0 & 1
\end{array}\right] \\
& A_{i, k}:=\left[\begin{array}{cc}
1+d_{t} \frac{7 \theta_{i, 1} \overline{\dot{m}}_{j, e, k}{ }^{\frac{3}{4}}}{4} & -d_{t} \theta_{i, 2} \\
d_{t} \theta_{i, 3} & 1
\end{array}\right] \\
& B_{i}:=\left[\begin{array}{ll}
d_{t} \theta_{i, 2} & 0
\end{array}\right]^{T} \\
& E_{i}:=\left[\begin{array}{ll}
0 & -d_{t} \theta_{i, 3}
\end{array}\right]^{T}
\end{aligned}
$$

With

$\theta_{i, 1}:=-0.316\left(\frac{4}{\pi D_{i} \mu}\right)^{-\frac{1}{4}} \frac{L_{i}}{D_{i}} \frac{1}{2 \rho V_{i}}, \theta_{i, 2}:=\frac{S_{i}^{2}}{V_{i}}, \theta_{i, 3}:=\frac{c^{2}}{V_{i}}$ and $i=1, \ldots, 3, j=4, \ldots, 6, \overline{\dot{m}}_{j, e, k}$ the input mass flow rate equilibrium trajectory.

The overall system and detection method is equivalent to three independent observers, one for each line.

The first objective is to design an observer depending only on known input and output measurements. We propose to use an EUIO with the following structure (Witczak, 2007):

$$
\left\{\begin{array}{c}
Z_{k+1}=N_{k+1} Z_{k}+K_{k+1} Y_{k}+G U_{k} \\
\hat{X}_{k+1}=Z_{k+1}+H Y_{k+1}
\end{array}\right.
$$

The above matrices are designed in such a way as to ensure unknown input decoupling as well as the minimization of the state estimate error.

$$
e_{k}=\hat{X}_{k}-X_{k}=Z_{k}-X_{k}+H Y_{k}
$$

To reduce its expression to a homogeneous equation we impose:

$$
\begin{aligned}
G= & T B \\
T A_{k}-N_{k+1} T-K_{k+1} C & =0 \\
T E & =0
\end{aligned}
$$

With $T=I_{n}-H C$ and $n$ the dimension of the state.

A necessary condition for the existence of a solution is $\operatorname{rank}(C E)=\operatorname{rank}(E)$. A particular solution is then:

$$
H=E\left((C E)^{T}(C E)\right)^{-1}(C E)^{T}
$$

The matrix $N_{k+1}$ should be Hurwitz to make the observer converge asymptotically.

This is the case if we choose:

$$
N_{k+1}=T A_{k}-K_{k+1} C
$$

The gain matrix $K_{k+1}$ is chosen to minimize the variance of the state estimation error. For a linear estimator under gaussian hypotheses, this translates into:

$$
K_{k+1}=T A_{k+1} P_{k} C^{T}\left(C P_{k} C^{T}-R_{k}\right)^{-1}
$$

The covariance matrix is then given by:

$$
\begin{aligned}
P_{k+1} & =T A_{k+1} P_{k} T A_{k+1}^{T}-K_{k+1} C P_{k} T A_{k+1}^{T} \\
& +H R_{k+1} H^{T}+T Q_{k} T^{T}
\end{aligned}
$$

The state estimation error (6) is taken as a residual.

\subsection{FDI with an adaptive CUSUM and EWMA-C shift estimator}

The FDI mechanism is supposed to detect any relevant failure that could lead to engine performance degradations. This shall be done sufficiently early to set up timely safe recovery. One way to proceed to detect faults is to evaluate the residual 
Table 1. Parameters for fault detection

\begin{tabular}{cc} 
Parameters & Values \\
\hline$\delta_{+, \text {min }}$ & $4 \mathrm{e}-2$ \\
$\delta_{-, \text {min }}$ & $-4 \mathrm{e}-2$ \\
$\lambda$ & 1.1055 \\
Threshold coefficient & $4.5 \mathrm{e} 4$ \\
\hline
\end{tabular}

corresponding to our state estimator error. The objective is then to be able to detect a residual mean shift for a nominal behavior, see (Basseville et al., 1993).

The two hypotheses considered are then:

H0: The mean value of the residual is nominal $\mu=\mu_{0}$ H1: The mean value of the residual has a shift $\mu=\mu_{1}$

In most common practical cases $\mu_{1}$ is unknown. This can be overcome by using Adaptive CUSUM (ACUSUM) algorithm which estimates this value as in (Jiang et al., 2008).

As mean shift amplitudes can vary drastically for a class of failure, the estimator designed for $\delta$ is a generalization of the Exponentially Weighted Moving Average (EWMA) which presents enhanced efficiency for estimation of large mean shifts under the form:

$$
\hat{\delta}_{k}=\hat{\delta}_{k-1}+\Phi_{\gamma}\left(e_{p, k}\right)
$$

With $e_{p, k}=r_{k}-\hat{\delta}_{k-1}$ the prediction error, $\Phi_{\gamma}$ is defined as a Huber score function.

$$
\Phi_{\gamma}:=\left\{\begin{array}{cc}
e_{p}+(1-\lambda) \gamma & , e_{p}<-\gamma \\
\lambda e_{p} & ,\left|e_{p}\right| \leq \gamma \\
e_{p}-(1-\lambda) \gamma & , e_{p}>\gamma
\end{array}\right.
$$

With $\gamma \geq 0$, usually fixed constant.

This leads to the following ACUSUM Statistic:

$$
s_{k}=\frac{ \pm\left|\hat{\delta}_{ \pm}\right|}{\sigma^{2}}\left(r_{k}-\mu_{0} \pm \frac{\left|\hat{\delta}_{ \pm}\right|}{2}\right)
$$

where for a mean shift increase or decrease:

$$
\hat{\delta}_{+}:=\max \left(\delta_{+, \min }, \hat{\delta}_{k}\right), \quad \hat{\delta}_{-}:=\min \left(\delta_{-, \min }, \hat{\delta}_{k}\right)
$$

$\delta_{+, \min }$ and $\delta_{-, \min }$ are here the minimum mean shifts amplitudes to detect (Table 1).

The threshold $\lambda$ is chosen to be a security coefficient times $\hat{\delta}_{+}$.

$\gamma$ is defined here at each step by $\gamma:=\left|r_{k}-\hat{\delta}_{k-1}\right| / 2$. With this choice of $\gamma$, the algorithm is more efficient for the detection of small shifts. This generalization is referred to as an EWMA-C statistic. To select the coefficients values and test the algorithm performance, three faults have been simulated with Carins for different profiles of the cooling circuit inflow valves closures and openings. The objective of this fault detection system is to be able to detect abrupt changes and to differentiate state perturbations from speed transients (first peak in the residuals - see Figures 4,5 and 6) characterized by slower variations than those of a failure.

The first fault simulated is an abrupt obstruction with a large mean shift on the line 1, the second is the same on the line 2 and the third one on the line 3 . It is sufficient to simulate faults in this part of the circuit since the method used will remain the same in the other part with 4 lines (see Figure 2). The total time of the simulation is 1090 seconds with a time step of 1 millisecond. The cadence of the estimation and the detection is 1 time step per 3 milliseconds (Table 2).

Table 2. Simulated faults

\begin{tabular}{ccc} 
Fault & $t_{\text {begin }}(m s)$ & $t_{\text {end }}(m s)$ \\
\hline Fault 1 & 651 & 1092 \\
Fault 2 & 651 & 1092 \\
Fault 3 & 678 & 1099 \\
\hline
\end{tabular}

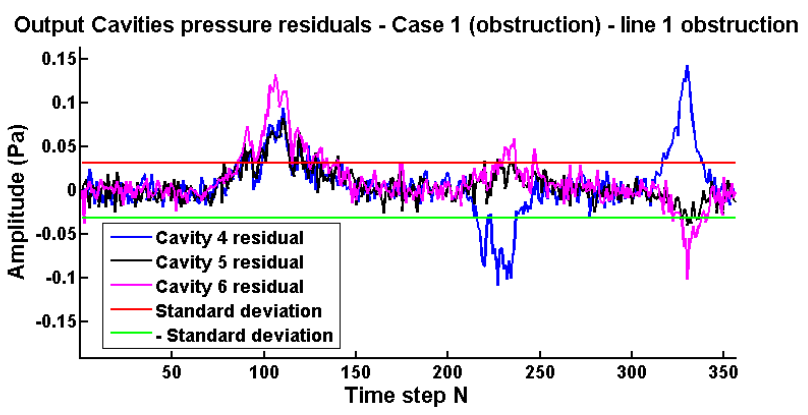

Figure 4. Pressure residual

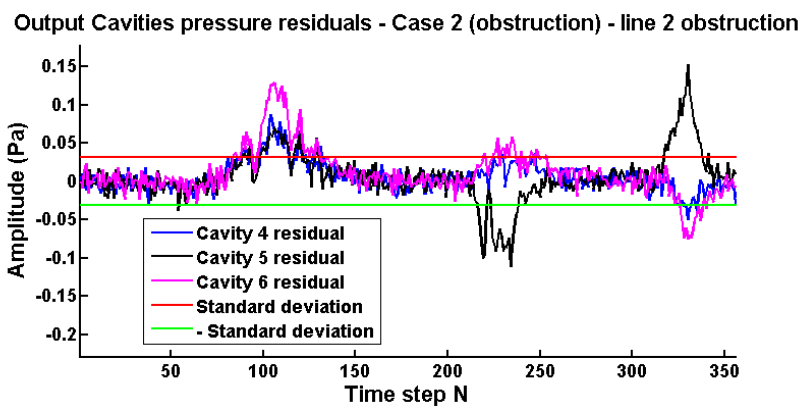

Figure 5. Pressure residual

The EUIO from the previous subsection permits to estimate outputs and generate the residual as the state estimator error defined by $r_{k}:=Y_{k}-C \hat{X}_{k}$ (Figures 4,5,6). After eliminating the effect of process input signals, filtering the effect of disturbances and model uncertainties on the residual, a residual evaluator has been designed by choosing an evaluation function and determining the threshold. 


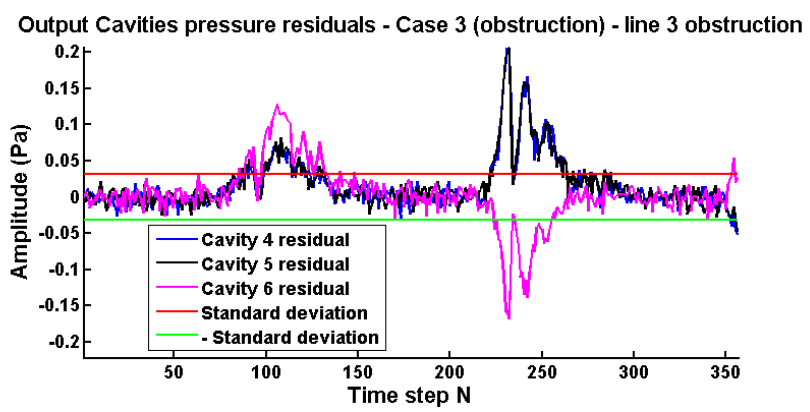

Figure 6. Pressure residual

\section{FAULT isOlation METHOD}

We consider in this part an additive actuator failure on the system. Once the fault has been detected by an on-line and real-time first fault detection mechanism the goal is to isolate the fault by a parity check.

To perform a parity check, we define the faulty system as:

$$
\left\{\begin{array}{c}
X_{k+1}=A_{k} X_{k}+B U_{k}+E D_{k}+F f_{k} \\
Y_{k+1}=C X_{k+1}
\end{array}\right.
$$

The fault distribution matrix could be different from the unknown input distribution matrix. In this case, the projection matrix for the parity test will remain of the same form but its coefficients will change. In the studied system (the cooling circuit) and for the type of simulated fault (an obstruction), those matrices are the same.

The previous balance equations can be augmented in order to define parity relations. After a linear dynamic transformation, these relations can be used for disturbance decoupling and isolation. Modeling the dynamics of our system during the transient phase requires to integrate time delays in the model. The fault dynamics for the next time step is not only determined by the current state but also by its former values. Considering these equations from time instant $k-L$ to time instant $k$ is a solution to overcome this problem and to ensure a temporal redundancy (over this window we assume the matrix $A_{k}$ to be constant in time):

$$
Y_{L, k}=A_{L} X_{k-L}+B_{L} U_{L}+E_{L}\left(D_{L}+f_{L}\right)
$$

Assuming $A_{L}:=\left[\begin{array}{c}C \\ C A \\ \ldots \\ C A^{L}\end{array}\right]$,
$B_{L}:=\left[\begin{array}{ccccc}0 & 0 & \ldots & 0 & 0 \\ C B & 0 & \ldots & 0 & 0 \\ \ldots & \ldots & \ldots & \ldots & \ldots \\ C A^{L-1} B & C A^{L-2} B & \ldots & C B & 0\end{array}\right]$
$E_{L}:=\left[\begin{array}{ccccc}0 & 0 & \ldots & 0 & 0 \\ C E & 0 & \ldots & 0 & 0 \\ \ldots & \dddot{\ldots} & \ldots & \ldots & \ldots \\ C A^{L-1} E & C A^{L-2} E & \ldots & C E & 0\end{array}\right]$.

The aim is to design a residual signal which is near zero in fault free case and non zero when a fault occurs in the monitored system. Then, for the parity check we search $H_{L}$ in such that:

$$
\begin{aligned}
& H_{L}\left(Y_{L}-B_{L} U_{L}-E_{L} D_{L}\right)=H_{L} A_{L} X_{k-L}+H_{L} E_{L} f_{L} \\
& H_{L}\left(Y_{L}-B_{L} U_{L}-E_{L} D_{L}\right)=H_{L} E_{L} f_{L}
\end{aligned}
$$

With $H_{L}$ the projection matrix. The projection matrix for the parity check can then be chosen by augmenting our previous system of equations with the following relations (20), (21), (22), (23). The parallel lines have to respect the mass flow rate continuity and the energy conservation. An obstruction in a line induces an increase of the mass flow rate in the other lines and a pressure drop in a line induces a pressure increase in the other lines.

The mass flow rate continuity gives:

$$
\dot{m}_{0, k}=\dot{m}_{1, k}+\dot{m}_{2, k}+\dot{m}_{3, k}
$$

We can then use Euler conservation equations for an incompressible fluid.

$$
\begin{aligned}
P_{i, k+1}-P_{i, k} & =-d_{t} \frac{c^{2}}{V_{i}}\left(\dot{m}_{i, k, e}-\dot{m}_{i, k, s}\right) \\
\dot{m}_{j, k+1, e}-\dot{m}_{j, k, e} & =-\frac{d_{t} S_{i}^{2}\left(P_{j, k}-P_{i, k}\right)}{V_{i}} \\
& +\frac{k_{p} d_{t} \dot{m}_{j, k, e}^{2}}{2 \rho V_{i}} \\
P_{j, k+1}-P_{j, k} & =-d_{t} \frac{c^{2}}{V_{i}}\left(\dot{m}_{j, k, s}-\dot{m}_{j, k, e}\right)
\end{aligned}
$$

for $i=1, \ldots, 3, j=4, \ldots, 6$.

This yields

$$
\begin{aligned}
\dot{m}_{0, k} & =\dot{m}_{4, k, e}+\frac{V_{1}\left(P_{1, k+1}-P_{1, k}\right)}{d_{t} c^{2}}+\dot{m}_{5, k, e} \\
& +\frac{V_{2}\left(P_{2, k+1}-P_{2, k}\right)}{d_{t} c^{2}}+\dot{m}_{6, k, e} \\
& +\frac{V_{3}\left(P_{3, k+1}-P_{3, k}\right)}{d_{t} c^{2}}
\end{aligned}
$$

For an established flow, with the residuals generated we can consider that the transients end at the 150th iteration. The detection algorithm is then activated after the transient to not consider them as failures in a first time.

Assuming that a failure impacts proportionally the mass flow rate: $\dot{m}_{j, k, e}:=\left(f_{r, i, k}+1\right) \dot{m}_{j, k, e, \text { nominal }}$ or again: 
$\dot{m}_{j, k, e}:=\left(f_{r, i, k}+1\right) \sqrt{\frac{2 S^{2}\left(\Delta P_{\text {nominal }}\right)}{k_{p}}-\frac{2 \rho V\left(\Delta \dot{m}_{e, n o m i n a l}\right)}{k_{p} d_{t}}}$.

We can write the expression of faults in each line $f_{r, i, k}$ for the case of a single fault and 2 simultaneous faults (full expressions are given in Appendix 1). With the help of those expressions we can then find the projection matrices. We have:

$$
Y_{k+1}-C B U_{k}-C E D_{k}=C E f_{k}+C A_{k} X_{k}
$$

Since $C B=0$, we have:

$$
\begin{gathered}
Y_{k+1}-C B U_{k}-C E D_{k}=Y_{k+1}-C E D_{k} \\
C E D_{k}=\left[\begin{array}{c}
-\frac{c^{2} d_{t}}{V_{1}} \dot{m}_{4, k, s} \\
-\frac{c^{2} d_{t}}{V_{2}} \dot{m}_{5, k, s} \\
-\frac{c^{2} d_{t}}{V_{3}} \dot{m}_{6, k, s}
\end{array}\right]
\end{gathered}
$$

and

$$
\dot{m}_{j, k, s}=\dot{m}_{j, k, e}-\frac{V_{i}\left(P_{j, k+1}-P_{j, k}\right)}{c^{2} d_{t}}
$$

for $i=1, \ldots, 3, j=4, \ldots, 6$. Then:

$$
\begin{aligned}
& Y_{k+1}-C E D_{k}= \\
& {\left[\begin{array}{c}
\frac{c^{2} d_{t}}{V_{1}}\left(\dot{m}_{0, k}-\dot{m}_{6, k, e}-\dot{m}_{5, k, e}\right)-\left(P_{1, k+1}-P_{1, k}\right) \\
-\frac{V_{2}\left(P_{2, k+1}-P_{2, k}\right)}{V_{1}}-\frac{V_{3}\left(P_{3, k+1}-P_{3, k}\right)}{V_{1}}+P_{4, k} \\
\frac{c^{2} d_{t}}{V_{2}}\left(\dot{m}_{0, k}-\dot{m}_{4, k, e}-\dot{m}_{6, k, e}\right)-\frac{V_{1}\left(P_{1, k+1}-P_{1, k}\right)}{V_{2}} \\
-\left(P_{2, k+1}-P_{2, k}\right)-\frac{V_{3}\left(P_{3, k+1}-P_{3, k}\right)}{V_{2}}+P_{5, k} \\
\frac{c^{2} d_{t}}{V_{3}}\left(\dot{m}_{0, k}-\dot{m}_{5, k, e}-\dot{m}_{4, k, e}\right)-\frac{V_{1}\left(P_{1, k+1}-P_{1, k}\right)}{V_{3}} \\
-\frac{V_{2}\left(P_{2, k+1}-P_{2, k}\right)}{V_{3}}-\left(P_{3, k+1}-P_{3, k}\right)+P_{6, k}
\end{array}\right]}
\end{aligned}
$$

With:

$$
\begin{aligned}
& \dot{m}_{j, k, e}= \\
& \sqrt{\frac{2 S_{i}^{2} \rho\left(P_{j, k}-P_{i, k}\right)}{k_{p}}-\frac{2 \rho V_{i}\left(\dot{m}_{j, k+1, e}-\dot{m}_{j, k, e}\right)}{k_{p} d_{t}}}
\end{aligned}
$$

for $i=1, \ldots, 3, j=4, \ldots, 6$. The projection matrix $H$ has to verify:

$$
H C A_{k} X_{k}=0
$$

Using (24), $H$ appears to be equal to:

$$
H:=\left[\begin{array}{lll}
h_{1} & h_{2} & h_{3} \\
h_{1} & h_{2} & h_{3} \\
h_{1} & h_{2} & h_{3}
\end{array}\right]
$$

With: $h_{i}:=\frac{\omega_{i, k}}{3 \frac{d_{t} c^{2}}{V_{i}} \dot{m}_{j, k, e}+3 P_{j, k}}, i=1 \ldots 3, j=4 \ldots 6$ and $\omega_{i, k}:=\dot{m}_{0, k}-3\left(\dot{m}_{j, k, e}-\frac{V_{1}\left(P_{i, k+1}-P_{i, k}\right)}{d_{t} c^{2}}\right)$
Since:

$$
\begin{aligned}
& P_{j, k}=P_{j, k+1}+\frac{d_{t} c^{2}}{V_{i}}\left(\dot{m}_{j, k, s}-\dot{m}_{j, k, e}\right) \\
& P_{j, k}=Y_{i, k+1}-\left(C E D_{k}\right)_{i}-\frac{d_{t} c^{2}}{V_{i}} \dot{m}_{j, k, e}
\end{aligned}
$$

for $i=1, \ldots, 3, j=4, \ldots, 6$.

Then:

$$
H_{L}:=\left[\begin{array}{cccc}
H & 0 & \ldots & 0 \\
0 & H & \ldots & 0 \\
\ldots & \ldots & \ldots & \ldots \\
0 & 0 & \ldots & H
\end{array}\right]_{L}
$$

The estimate of faults $f_{L}$ is then obtained from (see Figure 7 for results on the example):

$$
f_{L}=\left(H_{L} E_{L}\right)^{-1} H_{L}\left(Y_{L}-E_{L} D_{L}\right)
$$

To isolate the fault we can compare the variation of faults.

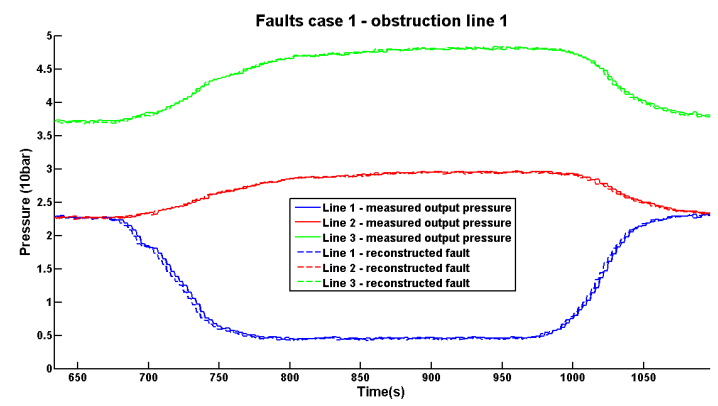

Figure 7. Case 1 reconstructed faults

-If the variation is of the same sign for two pipes and the residual of the third pipe is under the threshold fixed by the sum of the other pipes fault variations, the fault occurs in the first 2 pipes: an obstruction in two lines implies their mass flow rate decrease so that the mass flow rate continuity allow us to conclude that the mass flow rate increases in the last line. To differentiate the one fault case to the two faults case we can set a threshold based on the sum of the faults variations in the faulty lines (see equations (38) and (39)).

-If the variation is negative for two pipes then the fault occurs in the other pipe (one fault case): an obstruction implies a mass flow rate decrease in the impacted line so that the mass flow rate continuity for the overall system allow us to conclude that the mass flow rate increases in the other lines.

-While the sign of variations remain the same, faults are persisting.

This analysis is resumed in Tables 3 and 4. The signatures are given in Tables 6 and 7 . The terms $-{ }_{\max },+_{\max },-{ }_{\min },{ }_{\min }$ indicates if a fault in a line is of greater amplitude than the 
fault in the other line.The arrows indicates if the mean values of the residuals increase or decrease.

Table 3. Residuals variations - 1 failure cases

\begin{tabular}{cccc} 
Failure & Case 1 & Case 2 & Case 3 \\
\hline$r_{1}$ & $+/-$ & $\downarrow / \uparrow$ & $\downarrow / \uparrow$ \\
$r_{2}$ & $\downarrow / \uparrow$ & $+/-$ & $\downarrow / \uparrow$ \\
$r_{3}$ & $\downarrow / \uparrow$ & $\downarrow / \uparrow$ & $+/-$ \\
\hline
\end{tabular}

Table 4. Residuals variations - 2 failures cases

\begin{tabular}{|c|c|c|c|c|c|c|}
\hline Residuals & & & & Case 4 & & \\
\hline$r_{1}$ & + & - & $-\max$ & $+_{\max }$ & $+_{\text {min }}$ & $-{ }_{\text {min }}$ \\
\hline$r_{2}$ & + & - & $+_{\min }$ & ${ }^{-} \max$ & ${ }^{-} \max$ & $+_{\min }$ \\
\hline$r_{3}$ & $\downarrow$ & $\uparrow$ & 1 & $\downarrow$ & $\uparrow$ & \\
\hline Residuals & & & & Case 5 & & \\
\hline$r_{1}$ & + & - & $-\max$ & $+_{\max }$ & $+_{\min }$ & $-{ }_{\min }$ \\
\hline$r_{2}$ & $\downarrow$ & $\uparrow$ & / & $\downarrow$ & $\uparrow$ & / \\
\hline$r_{3}$ & + & - & $+_{\min }$ & ${ }^{-} \max$ & $-{ }_{\max }$ & $+_{\min }$ \\
\hline Residuals & & & & Case 6 & & \\
\hline$r_{1}$ & $\downarrow$ & $\uparrow$ & / & $\downarrow$ & $\uparrow$ & 1 \\
\hline$r_{2}$ & + & - & $-\max$ & $+_{\max }$ & $+_{\min }$ & $-\min$ \\
\hline$r_{3}$ & + & - & $+_{\min }$ & $-\max$ & $-{ }_{\max }$ & $+_{\min }$ \\
\hline
\end{tabular}

To evaluate the effectiveness of the designed algorithm, the good detection and false detection rates (GDR, FDR) have been calculated. For simultaneous faults we consider to be a good detection the simultaneous detection and localization of the faults in the two impacted lines, if at least one detection is false then we consider it to be a false detection.

-Case 1 : fault in line 1.

-Case 2 : fault in line 2.

-Case 3 : fault in line 3.

-Case 4 : fault in lines 1 and 2.

-Case 5 : fault in lines 1 and 3 .

-Case 6 : fault in lines 2 and 3 .

Those rates, which are satisfying, (Table 5) have been calculated from ten runs for each simulation and the settings have been chosen to optimize the good detection rate and minimize the false detection rate of abrupt mean shifts.

Table 5. Detection rates

\begin{tabular}{ccc} 
Fault & GDR & FDR \\
\hline Fault 1 & $96.06 \%$ & $0.00 \%$ \\
Fault 2 & $91.07 \%$ & $4.51 \%$ \\
Fault 3 & $90.40 \%$ & $4.51 \%$ \\
Fault 4 & $80.29 \%$ & $4.51 \%$ \\
Fault 5 & $93.06 \%$ & $3.75 \%$ \\
Fault 6 & $90.21 \%$ & $3.75 \%$ \\
\hline
\end{tabular}

Table 6. Isolability table - 1 failure cases

\begin{tabular}{cccc} 
Failure & Case 1 & Case 2 & Case 3 \\
\hline$f_{1}$ & 1 & 0 & 0 \\
$f_{2}$ & 0 & 1 & 0 \\
$f_{3}$ & 0 & 0 & 1 \\
\hline
\end{tabular}

Table 7. Isolability table - 2 failures cases

\begin{tabular}{cccc} 
Failure & Case 4 & Case 5 & Case 6 \\
\hline$f_{1}$ & 1 & 0 & 0 \\
$f_{2}$ & 0 & 1 & 0 \\
$f_{3}$ & 0 & 0 & 1 \\
\hline
\end{tabular}

\section{Conclusion}

In this paper a new model was proposed for the evolution of pressure and mass flow rates in the cooling circuit of a cryogenic test bench. This model has been validated on real data of the ONERA/CNES Mascotte test bench. Faults in the actuators are detected by the FDI method based on a fault estimator and an EUIO. This FDI method consists in a first detection with observer-based residual generation. Residuals are analyzed with the means of an ACUSUM. Then a parityspace based method has been proposed to isolate faults, using a projection matrix defined by dynamics fluid mechanic relations for the overall system.

Those methods have been tested with good results on simulations of the bench for different cases of failures, including simultaneous ones. This method permits to differentiate transients from failures and to detect failures during those transients if needed. Future work will address the design of a method to improve the performances during transients by fixing predetermined nominal trajectories. 


\section{REFERENCES}

Basseville, M., Nikiforov, I. V., et al. (1993). Detection of abrupt changes: theory and application (Vol. 104). Prentice Hall Englewood Cliffs.

Darouach, M., Zasadzinski, M., \& Xu, S. J. (1994). Fullorder observers for linear systems with unknown inputs. IEEE Transactions on Automatic Control, 39(3), 606-609.

Gao, Z., Cecati, C., \& Ding, S. X. (2015). A survey of fault diagnosis and fault-tolerant techniques part $\mathrm{i}$ : Fault diagnosis with model-based and signal-based approaches. IEEE Transactions on Industrial Electronics, 62(6), 3757-3767.

Gertler, J. (2017). Fault detection and diagnosis in engineering systems. Routledge.

Iannetti, A., Marzat, J., Piet-Lahanier, H., Ordonneau, G., \& Vingert, L. (2014). Development of model-based fault diagnosis algorithms for mascotte cryogenic test bench. In Journal of physics: Conference series (Vol. 570, p. 072006).

Jiang, W., Shu, L., \& Apley, D. W. (2008). Adaptive cusum procedures with ewma-based shift estimators. IIE Transactions, 40(10), 992-1003.

Kim, P. S., \& Lee, E. H. (2005). A new parity space approach to fault detection for general systems. In International conference on high performance computing and communications (pp. 535-540).

Nakayama, Y. (1998). Introduction to fluid mechanics. Butterworth-Heinemann.

Ryu, J.-H., Wan, H., \& Kim, S. (2010). Optimal design of a cusum chart for a mean shift of unknown size. Journal of Quality Technology, 42(3), 311-326.

Sarotte, C., Marzat, J., Piet-Lahanier, H., Iannetti, A., Galeotta, M., \& Ordonneau, G. (2018). Actuator fault tolerant system for cryogenic combustion bench cooling circuit. In 10th ifac symposium on fault detection, supervision and safety for technical processes safeprocess 2018.

Wang, H., Tian, Z., Shi, S., \& Weng, Z. (2010). Fault detection and isolation scheme based on parity space method for discrete time-delay system. In Fault detection. InTech.

Witczak, M. (2007). Modelling and estimation strategies for fault diagnosis of non-linear systems: from analytical to soft computing approaches (Vol. 354). Springer Science \& Business Media.

Zhong, M., Song, Y., \& Ding, S. X. (2015). Parity spacebased fault detection for linear discrete time-varying systems with unknown input. Automatica, 59, 120126.

\section{APPENDIX}

In the case of an obstruction in the line 1:

$$
\begin{aligned}
f_{r, 1, k} & =\left(\frac{\dot{m}_{0, k}}{\sqrt{\rho}}-\frac{c^{2}}{V_{2 \sqrt{\rho}}}\left(P_{2, k+1}-P_{2, k}\right)\right. \\
& -\sqrt{\frac{2 V_{2}}{k_{p} d_{t}}\left(d_{t} \frac{S_{2}^{2}}{V_{2}} \Delta P_{5,2, k}+\left(\dot{m}_{5, k+1, e}-\dot{m}_{5, k, e}\right)\right)} \\
& -\sqrt{\frac{2 V_{3}}{k_{p} d_{t}}\left(d_{t} \frac{S_{3}^{2}}{V_{3}} \Delta P_{6,3, k}+\left(\dot{m}_{6, k+1, e}-\dot{m}_{6, k, e}\right)\right)} \\
& -\frac{c^{2}}{V_{1} \sqrt{\rho}}\left(P_{1, k+1}-P_{1, k}\right) \\
& \left.-\frac{c^{2}}{V_{3 \sqrt{\rho}}}\left(P_{3, k+1}-P_{3, k}\right)\right) \\
& \frac{1}{\sqrt{\frac{2 S_{1}^{2} \Delta P_{4,1, n, k}}{k_{p}}+\frac{2 V_{1}\left(\dot{m}_{\left.4, k+1, e, n-\dot{m}_{4, k, e, n}\right)}\right.}{k_{p} d_{t}}}}-1
\end{aligned}
$$

$$
\begin{aligned}
f_{r, 2, k} & =\left(\frac{\dot{m}_{0, k}}{\sqrt{\rho}}-\frac{c^{2}}{V_{2} \sqrt{\rho}}\left(P_{2, k+1}-P_{2, k}\right)-\left(f_{r, 1}+1\right)\right. \\
& \sqrt{\frac{2 S_{1}^{2} \Delta P_{4,1, n, k}}{k_{p}}+\frac{2 V_{1}\left(\dot{m}_{4, k+1, e, n}-\dot{m}_{4, k, e, n}\right)}{k_{p} d_{t}}} \\
& -\sqrt{\frac{2 V_{3}}{k_{p} d_{t}}\left(d_{t} \frac{S_{3}^{2}}{V_{3}} \Delta P_{6,3, k}+\left(\dot{m}_{6, k+1, e}-\dot{m}_{6, k, e}\right)\right)} \\
& -\frac{c^{2}}{V_{1} \sqrt{\rho}}\left(P_{1, k+1}-P_{1, k}\right) \\
& \left.-\frac{c^{2}}{V_{3} \sqrt{\rho}}\left(P_{3, k+1}-P_{3, k}\right)\right) \\
& \frac{1}{\sqrt{\frac{2 S_{2}^{2} \Delta P_{5,2, n, k}}{k_{p}}+\frac{2 V_{2}\left(\dot{m}_{5, k+1, n, e}-\dot{m}_{5, k, e, n}\right)}{k_{p} d_{t}}}}-1
\end{aligned}
$$

$$
\begin{aligned}
f_{r, 3, k} & =\left(\frac{\dot{m}_{0, k}}{\sqrt{\rho}}-\frac{c^{2}}{V_{2} \sqrt{\rho}}\left(P_{2, k+1}-P_{2, k}\right)-\left(f_{r, 1}+1\right)\right. \\
& \sqrt{\frac{2 S_{1}^{2} \Delta P_{4,1, n, k}}{k_{p}}+\frac{2 V_{1}\left(\dot{m}_{4, k+1, e, n}-\dot{m}_{4, k, e, n}\right)}{k_{p} d_{t}}} \\
& -\sqrt{\frac{2 V_{2}}{k_{p} d_{t}}\left(d_{t} \frac{S_{2}^{2}}{V_{2}} \Delta P_{5,2, k}+\left(\dot{m}_{5, k+1, e}-\dot{m}_{5, k, e}\right)\right)} \\
& -\frac{c^{2}}{V_{1} \sqrt{\rho}}\left(P_{1, k+1}-P_{1, k}\right) \\
& \left.-\frac{c^{2}}{V_{3} \sqrt{\rho}}\left(P_{3, k+1}-P_{3, k}\right)\right) \\
& \frac{1}{\sqrt{\frac{2 S_{3}^{2} \Delta P_{6,3, n, k}}{k_{p}}+\frac{2 V_{3}\left(\dot{m}_{6, k+1, e, n}-\dot{m}_{6, k, e, n}\right)}{k_{p} d_{t}}}}-1
\end{aligned}
$$


In the case of 2 failures, for example in lines 1 and 2 :

$$
\begin{aligned}
& \left(f_{r, 1, k}+1\right) \\
& \sqrt{\frac{2 S_{1}^{2} \Delta P_{4,1, n, k}}{k_{p}}+\frac{2 V_{1}\left(\dot{m}_{4, k+1, e, n}-\dot{m}_{4, k, e, n}\right)}{k_{p} d_{t}}} \\
& +\left(f_{r, 2, k}+1\right) \\
& \sqrt{\frac{2 S_{2}^{2} \Delta P_{5,2, n, k}}{k_{p}}+\frac{2 V_{2}\left(\dot{m}_{5, k+1, e, n}-\dot{m}_{5, k, e, n}\right)}{k_{p} d_{t}}} \\
& =\frac{\dot{m}_{0, k}}{\sqrt{\rho}}-\frac{c^{2}}{V_{2} \sqrt{\rho}}\left(P_{2, k+1}-P_{2, k}\right) \\
& -\sqrt{\frac{2 V_{3}}{k_{p} d_{t}}\left(d_{t} \frac{S_{3}^{2}}{V_{3}} \Delta P_{6,3, k}+\left(\dot{m}_{6, k+1, e}-\dot{m}_{6, k, e}\right)\right)} \\
& -\frac{c^{2}}{V_{1} \sqrt{\rho}}\left(P_{1, k+1}-P_{1, k}\right) \\
& -\frac{c^{2}}{V_{3 \sqrt{\rho}}}\left(P_{3, k+1}-P_{3, k}\right)
\end{aligned}
$$

$$
\begin{aligned}
f_{r, 3, k} & =\left(\frac{\dot{m}_{0, k}}{\sqrt{\rho}}-\frac{c^{2}}{V_{2} \sqrt{\rho}}\left(P_{2, k+1}-P_{2, k}\right)\right. \\
& -\left(f_{r, 1, k}+1\right) \\
& \sqrt{\frac{2 S_{1}^{2} \Delta P_{4,1, n, k}}{k_{p}}+\frac{2 V_{1}\left(\dot{m}_{4, k+1, e, n}-\dot{m}_{4, k, e, n}\right)}{k_{p} d_{t}}} \\
& -\left(f_{r, 2, k}+1\right) \\
& \sqrt{\frac{2 S_{2}^{2} \Delta P_{5,2, n, k}}{k_{p}}+\frac{2 V_{2}\left(\dot{m}_{5, k+1, e, n}-\dot{m}_{5, k, e, n}\right)}{k_{p} d_{t}}} \\
& -\frac{c^{2}}{V_{1} \sqrt{\rho}}\left(P_{1, k+1}-P_{1, k}\right) \\
& \left.-\frac{c^{2}}{V_{3} \sqrt{\rho}}\left(P_{3, k+1}-P_{3, k}\right)\right) \\
& \sqrt{\frac{2 S_{3}^{2} \Delta P_{6,3, n, k}}{k_{p}}+\frac{2 V_{3}\left(\dot{m}_{6, k+1, e, n}-\dot{m}_{6, k, e, n}\right)}{k_{p} d_{t}}}
\end{aligned}
$$

